\title{
A multicenter cross-sectional survey of the knowledge, attitudes, and practices of geriatric nurses regarding dysphagia care
}

\author{
Chun-Rong Luo ${ }^{1}$, Jing-Yi Wei ${ }^{2}$, Xue-Mei Zhang ${ }^{3}$ \\ ${ }^{1}$ West China School of Nursing, Sichuan University/Operating Room, West China Hospital, Sichuan University, Chengdu, China; ${ }^{2}$ West China \\ School of Nursing, Sichuan University/The Center of Gerontology and Geriatrics, Sichuan University West China Hospital, Chengdu, China; ${ }^{3}$ The \\ Center of Gerontology and Geriatrics, Sichuan University West China Hospital, Chengdu, China \\ Contributions: (I) Conception and design: CR Luo; (II) Administrative support: XM Zhang; (III) Provision of study materials or patients: XM Zhang; \\ (IV) Collection and assembly of data: JY Wei; (V) Data analysis and interpretation: CR Luo, JY Wei; (VI) Manuscript writing: All authors; (VII) Final \\ approval of manuscript: All authors. \\ Correspondence to: Xue-Mei Zhang. 37 Guoxue Alley, Wuhou District, Chengdu 610041, China. Email: zxm0709@126.com.
}

\begin{abstract}
Background Dysphagia is common in the elderly population, and it can lead to complications such as aspiration, undernutrition, and psychological and social interaction disorders in the elderly and have a great impact on quality of life. This study aimed to understand the current knowledge, attitudes, and practices (KAP) of geriatric nurses regarding dysphagia care for elderly adults and to analyze the relevant influencing factors to improve care plans for elderly patients with dysphagia.

Methods: A multicenter cross-sectional survey of 782 geriatric nurses from 17 hospitals in Sichuan Province was conducted using a questionnaire to determine their KAP regarding dysphagia care.

Results: The geriatric nurses' mean score for geriatric dysphagia-related knowledge was $55.30 \pm 0.61$ (out of a total score of 100). The results of a univariate analysis showed that the geriatric dysphagia-related knowledge scores differed significantly among the nurses according to their age, education level, title, hospital level, and years of work experience $(\mathrm{P}<0.05)$. The multivariate regression results indicated that title, hospital level, and experience with caring for elderly patients with dysphagia were the main factors that influenced geriatric dysphagia-related knowledge scores.

Conclusions: The geriatric nurses had acceptable practices and attitudes regarding dysphagia care, but their knowledge regarding geriatric dysphagia needs to be enhanced. Hospital administrators should provide targeted training to improve geriatric nurses' competency in dysphagia care and thus ensure the quality and safety of dysphagia care for elderly patients.
\end{abstract}

Keywords: Geriatric nurses; dysphagia; knowledge, attitudes, and practices (KAP)

Submitted Sep 01, 2021. Accepted for publication Jan 10, 2022.

doi: 10.21037/apm-21-3672

View this article at: https://dx.doi.org/10.21037/apm-21-3672

\section{Introduction}

Dysphagia refers to the inability to safely and effectively ingest food through the mouth or transport solid or liquid food to the stomach through the esophagus (1), which often leads to aspiration, undernutrition, and psychological and social interaction disorders and may have adverse consequences, such as death $(2,3)$. Aging, disease, and drugs are among the risk factors for the development of dysphagia. Compared with other populations, elderly adults are more susceptible to dysphagia. Relevant studies suggest that the incidence of dysphagia is $13-38 \%$ in communitydwelling elderly adults $(4,5)$ and $15-68 \%$ among elderly nursing home populations $(6,7)$. However, the incidence of dysphagia can reach $30-55 \%$ in hospitalized elderly patients due to risk factors such as multimorbidity and the simultaneous use of multiple drugs (8). Dysphagia has gradually become a common clinical problem among elderly adults. However, due to insufficient understanding 
of geriatric dysphagia, clinical attention to geriatric dysphagia is inadequate, which can lead to various adverse consequences. Previous research has shown that early and effective nursing intervention can reduce the occurrence of dysphagia-related complications. Therefore, the identification, assessment and intervention of dysphagia should be valued in clinical nursing. Nurses, as sentinels of medical services, have important responsibilities, such as monitoring, supervising, and educating patients as part of patient management. According to the theory of knowledge, attitudes and practices (KAP), knowledge and attitude have an impact on practice. Additionally, good attitudes and practices require a wealth of knowledge as a foundation. Therefore, the geriatric dysphagia-related knowledge of nurses, especially geriatric nurses who interact closely with the elderly, will determine the quality of dysphagia care that elderly patients receive. At present, foreign studies have reported that in view of the impact of swallowing intervention on the nutritional status and hydration status of patients, relevant training and assessment of clinical nursing staff should be further carried out (9). Previous studies on the KAP of nurses regarding the management of geriatric dysphagia have rarely included geriatric nurses; instead, they have focused primarily on neurology nurses. Furthermore, they have primarily investigated samples from tertiary general hospitals and have rarely included samples from secondary hospitals. Nurses from hospitals of different levels have different opportunities to receive training courses, participate in academic forums, and receive continuing education at other hospitals or institutions; consequently, their KAP situations differ. Therefore, secondary hospitals should be included to allow a unified analysis. In this study, 17 tertiary and secondary hospitals in Sichuan Province were included to analyze the $\mathrm{KAP}$ of geriatric nurses regarding dysphagia management and determine the influencing factors. We present the following article in accordance with the SURGE reporting checklist (available at https://apm.amegroups.com/article/ view/10.21037/apm-21-3672/rc).

\section{Methods}

\section{Survey tools}

At present, some researchers have developed a scale for nurses with dysphagia after stroke (10), but it is not applicable to geriatric nurses. Most studies of nurses' KAP of dysphagia were conducted with a self-made scale (questionnaire). The questionnaire was developed based on the "Expert Consensus on the Assessment and Treatment of Dysphagia in China" (2017 version) and the questionnaire on the knowledge of dysphagia management developed by Rhoda et al. (11). The questionnaire used in this study consisted of four subquestionnaires: (I) a subquestionnaire on general data (including the nurses' educational background, hospital type, hospital level, etc.), (II) a subquestionnaire on the geriatric nurses' dysphagiarelated knowledge (a total of 20 multiple choice questions; incorrect answers were scored as 0 , and correct answers were scored as 5; total scores ranged from $0-100$, with higher scores indicating better knowledge mastery), (III) a subquestionnaire on the geriatric nurses' attitudes toward dysphagia care (a total of 5 items rated with a 5 -point Likert scoring system corresponding to "completely disagree, disagree, neither agree nor disagree, agree, or completely agree"; higher scores indicated more positive attitudes toward dysphagia management), and (IV) a subquestionnaire on the geriatric nurses' practices regarding dysphagia management in elderly patients (a total of 6 items rated with a 5 -point Likert scale with responses from 1 to 5 corresponding to "never, sometimes, half of the time, most of the time, or always", respectively; higher scores indicated better dysphagia management practices). The higher the score of the questionnaire, the stronger the nursing ability of the geriatric nurses for swallowing disorders, and vice versa. A preliminary experiment was conducted before the formal survey, and unclear items were revised and sent to six experts with a rank of associate or higher to produce the formal questionnaire. The results of the preliminary experiment and the expert evaluation showed that the questionnaire had an internal consistency reliability (Cronbach's $\alpha$ coefficient) of 0.913 , a content validity of 0.956 , and a test-retest reliability of 0.933 .

\section{Survey subjects}

In this study, a total of 17 hospitals (14 tertiary hospitals and three secondary hospitals) from eastern, western, southern, northern, and central Sichuan Province were selected. The study lasted from April to May 2019. The nurses included in this study met the following criteria: the possession of a nurse's practice certificate; $\geq 3$ years of work experience in geriatrics; voluntary participation in the survey; and being on the job during the survey period. Exclusion criteria were as follows: nurses from other hospitals who were 
being trained at the study hospitals, and nurses who were receiving standardized training. This study was approved by the Ethics Committee of the West China Hospital of Sichuan University (No. 1123, approved in 2021) and conducted in accordance with the Declaration of Helsinki (as revised in 2013). All nurses gave their informed consent. According to the literature review and the second edition of Medical Statistics, edited by Zhenqiu Sun, a sample size of at least 15-20 times the number of variables included in the multivariate linear regression equation was required. A total of 29 independent variables were included in this survey. Taking into account a loss to follow-up rate of $20 \%$, the calculated sample size was 543-725 cases. To ensure a sufficient and effective sample size, 782 questionnaires were distributed during the survey process.

\section{Survey method}

The researchers contacted the head nurses of the geriatrics departments of the participating hospitals, explained the purpose and significance of the study, and provided training regarding the investigation methods to enable the head nurses to cooperate with the survey. The head nurse of the geriatrics department of each participating hospital was the person in charge for that hospital, and she delivered uniform instructions to explain the study to the survey subjects. After the survey subjects provided informed consent, an online survey was conducted in the form of electronic questionnaire. To ensure the authenticity of the study subjects' answers, the subjects were informed that their responses would be anonymous. To ensure the completeness and validity of questionnaires, all questions had to be answered before the questionnaire could be submitted, and only one questionnaire could be submitted per IP address.

\section{Statistical analysis}

The data were imported into SPSS 22.0 statistical software for statistical analysis. The general data of the study subjects are described as frequencies and percentages (\%). The mean score and accuracy rate of each knowledge dimension and the accuracy rate for each item are expressed as the mean \pm standard deviation $\left(\bar{x}_{ \pm s}\right)$ and percentage (\%). The means of two groups were compared using the independent-samples $t$-test. The means of multiple groups were compared using one-way analysis of variance. $\mathrm{P}<0.05$ was considered statistically significant.

\section{Results}

\section{Univariate analysis of the effect of geriatric nurses' general characteristics on their KAP regarding dysphagia care}

A total of 782 valid questionnaires (100\%) were collected in this survey, including 497 (63.6\%) from tertiary hospitals and 285 (36.4\%) from secondary hospitals. Most of the nurses $(61.0 \%)$ worked at general hospitals. A total of $59.5 \%$ of the nurses were $\leq 30$ years old, $50.4 \%$ of the nurses had $\leq 5$ years of work experience, $50.4 \%$ of the nurses had a bachelor's degree, $47.2 \%$ of the nurses were nurse practitioners, and $74.4 \%$ of the nurses had experience with caring for elderly patients with dysphagia. The results of the univariate analysis (Table 1) showed that the dysphagiarelated knowledge scores differed significantly among nurses according to their age, education level, hospital type, title, hospital level, and years of work experience $(\mathrm{P}<0.05)$ and that the practice and attitude scores differed significantly among nurses according to the type of hospital where they worked and the duration since they had last received training about dysphagia $(\mathrm{P}<0.05)$.

\section{Geriatric dysphagia-related knowledge scores of the geriatric nurses}

The geriatric nurses had geriatric dysphagia-related knowledge scores of $55.30 \pm 0.61$. The mean scores and accuracy rates for the items in each dimension are shown in Table 2. The three items with the lowest and highest accuracy rates are shown in Table 3.

\section{Results of the multivariate analysis of geriatric dysphagia-related knowledge among geriatric nurses}

Six items (age, hospital level, hospital type, years of work experience, education level, and title) had a positive association in the univariate analysis and were included as independent variables $(\mathrm{P}<0.05)$. Previous experience with caring for elderly patients with dysphagia was included as a seventh independent variable, and the geriatric dysphagiarelated knowledge score was used as the dependent variable in the multiple linear regression analysis (criteria: probability of $\mathrm{F}$ to enter $\leq 0.05$, probability of $\mathrm{F}$ to remove $\geq 0.10$ ). The main factors influencing nurses' geriatric dysphagia-related knowledge scores were their title, hospital level, and previous experience with caring for elderly patients with dysphagia. The independent variable assignment methods and the results of the analysis are 
Table 1 Univariate analysis of the relationship between the general characteristics of geriatric nurses and their knowledge, attitudes and practices toward dysphagia care $\left(\mathrm{n}=782, \bar{x}_{ \pm s}\right)$

\begin{tabular}{|c|c|c|c|c|c|}
\hline Item & Number of nurses & Proportion of nurses (\%) & Knowledge score & Attitude score & Practice score \\
\hline$\leq 30$ & 465 & 59.5 & $52.35 \pm 16.10$ & $20.25 \pm 4.04$ & $23.85 \pm 4.08$ \\
\hline $31-35$ & 169 & 21.6 & $58.20 \pm 16.94$ & $20.33 \pm 4.19$ & $24.38 \pm 3.48$ \\
\hline $36-40$ & 70 & 9.0 & $60.21 \pm 16.14$ & $21.03 \pm 3.62$ & $23.73 \pm 3.29$ \\
\hline$\geq 46$ & 46 & 5.9 & $62.28 \pm 15.33$ & $21.46 \pm 2.38$ & $23.48 \pm 3.13$ \\
\hline$t / F$ value & & & 9.886 & 1.516 & 2.298 \\
\hline$P$ value & & & $<0.001^{\star \star \star}$ & 0.196 & 0.057 \\
\hline \multicolumn{6}{|l|}{ Hospital level } \\
\hline Tertiary Class C & 2 & 0.3 & $42.50 \pm 10.61$ & $22.50 \pm 3.54$ & $24.00 \pm 8.49$ \\
\hline Secondary Class A & 173 & 22.1 & $51.04 \pm 15.44$ & $19.78 \pm 4.00$ & $23.14 \pm 4.05$ \\
\hline Secondary Class B & 111 & 14.2 & $52.75 \pm 15.61$ & $20.30 \pm 3.82$ & $24.27 \pm 4.24$ \\
\hline Secondary Class C & 1 & 0.1 & $30.00 \pm 00.00$ & $20.00 \pm 00.00$ & $24.00 \pm 00.00$ \\
\hline$t / F$ value & & & 11.380 & 1.525 & 2.041 \\
\hline$P$ value & & & $<0.001^{\star \star \star}$ & 0.179 & 0.071 \\
\hline \multicolumn{6}{|l|}{ Hospital type } \\
\hline General hospital & 477 & 61.0 & $57.95 \pm 17.20$ & $20.67 \pm 3.90$ & $23.88 \pm 3.60$ \\
\hline$\leq 5$ & 394 & 50.4 & $52.60 \pm 16.80$ & $20.28 \pm 3.93$ & $23.81 \pm 4.00$ \\
\hline $6-10$ & 230 & 29.4 & $55.83 \pm 15.73$ & $20.29 \pm 4.16$ & $24.00 \pm 3.89$ \\
\hline $11-15$ & 86 & 11.0 & $58.55 \pm 17.47$ & $20.81 \pm 3.69$ & $23.94 \pm 3.47$ \\
\hline$\geq 16$ & 72 & 9.2 & $64.51 \pm 16.87$ & $20.90 \pm 3.29$ & $23.67 \pm 3.15$ \\
\hline$t / F$ value & & & 12.074 & 0.892 & 0.204 \\
\hline$P$ value & & & $<0.001^{\star \star \star}$ & 0.445 & 0.894 \\
\hline \multicolumn{6}{|l|}{ Education level } \\
\hline Technical secondary school & 22 & 2.8 & $52.05 \pm 17.71$ & $19.77 \pm 3.89$ & $23.36 \pm 4.68$ \\
\hline Junior college & 364 & 46.5 & $51.47 \pm 16.30$ & $20.13 \pm 3.87$ & $23.73 \pm 3.90$ \\
\hline Bachelor's degree & 394 & 50.4 & $58.92 \pm 16.64$ & $20.67 \pm 3.96$ & $24.02 \pm 3.73$ \\
\hline
\end{tabular}

Table 1 (continued) 
Table 1 (continued)

\begin{tabular}{|c|c|c|c|c|c|}
\hline Item & Number of nurses & Proportion of nurses (\%) & Knowledge score & Attitude score & Practice score \\
\hline$t / F$ value & & & 14.075 & 1.811 & 0.567 \\
\hline$P$ value & & & $<0.001^{\star \star \star}$ & 0.144 & 0.637 \\
\hline \multicolumn{6}{|l|}{ Title } \\
\hline Nurse practitioner & 369 & 47.2 & $55.73 \pm 15.57$ & $20.48 \pm 3.83$ & $24.12 \pm 3.56$ \\
\hline Supervising nurse & 144 & 18.4 & $63.47 \pm 16.83$ & $20.81 \pm 3.50$ & $23.64 \pm 3.04$ \\
\hline Deputy head nurse & 22 & 2.8 & $66.14 \pm 15.43$ & $21.68 \pm 2.63$ & $23.27 \pm 3.52$ \\
\hline Head nurse & 3 & 0.4 & $48.33 \pm 2.89$ & $21.67 \pm 2.08$ & $23.33 \pm 1.53$ \\
\hline \multicolumn{6}{|c|}{ Duration since last dysphagia training } \\
\hline Less than one month & 147 & 18.8 & $53.33 \pm 18.60$ & $20.24 \pm 4.59$ & $24.07 \pm 4.94$ \\
\hline Less than half a year & 253 & 32.4 & $56.92 \pm 16.94$ & $20.41 \pm 4.41$ & $24.12 \pm 3.73$ \\
\hline Within one year & 144 & 18.4 & $55.63 \pm 16.62$ & $20.89 \pm 3.16$ & $24.42 \pm 3.15$ \\
\hline Within two years & 60 & 7.7 & $57.92 \pm 14.77$ & $20.47 \pm 3.33$ & $23.30 \pm 2.84$ \\
\hline More than two years ago & 178 & 22.8 & $53.48 \pm 16.17$ & $20.10 \pm 3.26$ & $23.08 \pm 3.61$ \\
\hline$t / F$ value & & & 1.968 & 0.879 & 3.399 \\
\hline$P$ value & & & 0.098 & 0.476 & $0.009^{\star \star}$ \\
\hline
\end{tabular}

${ }^{\Delta}, t$ value; ${ }^{*}, \mathrm{P}<0.05 ;{ }^{* *}, \mathrm{P}<0.01 ;{ }^{* *}, \mathrm{P}<0.001$

Table 2 Mean scores and accuracy rates of geriatric nurses on geriatric dysphagia-related knowledge items

\begin{tabular}{lcc}
\hline Dimension & Mean score for each dimension $(x \pm \mathrm{s})$ & Accuracy rate $(\%)$ \\
\hline Risk factors & $10.75 \pm 5.53$ & 43.02 \\
Clinical manifestations & $14.88 \pm 6.13$ & 59.51 \\
Assessment methods & $16.28 \pm 6.17$ & 65.12 \\
Intervention measures & $13.39 \pm 6.78$ & 53.56 \\
\hline
\end{tabular}


Table 3 The three geriatric dysphagia-related knowledge items with the lowest and highest accuracy rates $(\mathrm{n}=782)$

\begin{tabular}{l}
\hline Item \\
\hline The three items with the lowest accuracy rates \\
Cholinergic drugs (nicotine, etc.) do not affect swallowing function \\
Aspiration pneumonia can be caused by the entry of food and/or oral secretions into the airways and lungs \\
A decreased oral transit time is not the cause of dysphagia \\
The three items with the highest accuracy rates \\
Excretion status is not the main method for assessing dysphagia \\
Increased food intake is not a common clinical manifestation of dysphagia \\
The assessment methods for dysphagia do not include gastroscopy
\end{tabular}

Table 4 Independent variable assignment

\begin{tabular}{|c|c|}
\hline Independent variable & Assignment method \\
\hline Hospital level & $\begin{array}{l}\text { Tertiary Class } A=6 \text {, Tertiary Class } B=5 \text {, Tertiary Class } C=4 \text {, Secondary Class } A=3 \text {, } \\
\text { Secondary Class } B=2 \text {, Secondary Class } C=1\end{array}$ \\
\hline Hospital type & Geriatric hospital $=3$, General hospital $=2$, Other $=1$ \\
\hline Title & Nurse $=1$, Nurse practitioner $=2$, Supervising nurse $=3$, Deputy head nurse $=4$, Head nurse $=5$ \\
\hline $\begin{array}{l}\text { Previous experience with caring for } \\
\text { elderly adults with dysphagia }\end{array}$ & Yes $=1$, No $=0$ \\
\hline
\end{tabular}

shown in Table 4 and Table 5, respectively.

\section{Discussion}

\section{Nurses' knowledge of drug-related risk factors urgently needs to be strengthened}

Experts in dysphagia research suggest that the tendency toward frailty in the elderly population should be fully considered and should be included in routine screenings for dysphagia. The need for dysphagia management should be determined according to the preliminary screening results. The prerequisite for successful dysphagia screening in the elderly population is for nurses to have a good understanding of the risk factors for dysphagia. The results of this study showed that $43.02 \%$ of the nurses had accurate knowledge of the risk factors for dysphagia in elderly adults and that only $16.37 \%$ of the nurses correctly answered the item "cholinergic drugs (nicotine, etc.) do not affect swallowing function", which was the item with the lowest accuracy rate. Most of the nurses failed to accurately identify the types of drugs that have potential negative effects on the swallowing function of the elderly, which was consistent with the findings of Kelly et al. (12) that nurses had high error rates in the medication management of patients with dysphagia. In fact, certain types of drugs, such as sedatives and hypnotics, anticholinergics, and antihistamines, can adversely affect the swallowing function of elderly individuals. Sedatives and hypnotics can trigger extrapyramidal reactions and cause dystonia by reducing the excitability of the central nervous system, which leads to decreased coordination between oral processing and swallowing and thus affects eating. Antihistamines and anticholinergics may affect swallowing function by affecting saliva secretion. It is suggested that administrators pay attention to training geriatric nurses in the identification 
Table 5 Multivariate analysis of factors affecting the geriatric dysphagia-related knowledge of geriatric nurses $(\mathrm{n}=782)$

\begin{tabular}{|c|c|c|c|c|c|}
\hline Independent variable & $\begin{array}{l}\text { Nonstandardized } \\
\text { regression coefficient }\end{array}$ & Standard error & $\begin{array}{c}\text { Standardized regression } \\
\text { coefficient }\end{array}$ & $t$ value & $P$ value \\
\hline Constant & 27.583 & 6.173 & - & 4.468 & $<0.001$ \\
\hline Hospital level & 1.503 & 0.496 & 0.135 & 3.028 & 0.003 \\
\hline
\end{tabular}

of therapeutic drugs that may cause dysphagia in elderly patients to ensure that these nurses have sufficient knowledge to successfully perform routine screenings of populations at high risk for dysphagia.

\section{Geriatric nurses' knowledge for identifying and preventing aspiration needs to be enbanced}

In most cases, dysphagia occurs insidiously, especially in the elderly. In clinical practice, due to the nature of their job, nurses are the first professionals to identify the symptoms and signs of dysphagia in patients. Therefore, the accurate and timely identification of dysphagia symptoms is critical for clinical nurses, especially geriatric nurses. In this study, only $59.51 \%$ of the geriatric nurses had accurate knowledge of the clinical manifestations and complications of dysphagia, and only $27.88 \%$ correctly answered the item "aspiration pneumonia can be triggered by the entry of food and/or oral secretions into the airways and lungs", indicating that $72.12 \%$ of the geriatric nurses were unsure of the relationship between aspiration and pneumonia. The most common complication of dysphagia is aspiration. Aspiration is divided into silent aspiration and overt aspiration. Silent aspiration is caused by the infiltration of food, fluids or saliva into the subglottis without causing cough. Studies have shown that $15.6 \%$ of aspiration pneumonia is caused by silent aspiration (13) and that silent aspiration-induced pneumonia is associated with a higher mortality rate within one month (14). Therefore, the identification of aspiration, especially silent aspiration, is critical for geriatric nurses. In nursing practice, nurses should choose their favorite food to make paste according to the patient's dietary preferences to prevent the occurrence of aspiration. Administrators need to regularly organize opportunities for geriatric nurses to learn the information and methods associated with dysphagia identification and observation, especially certain high-risk complications of dysphagia, to achieve the early management of suspicious patients.

\section{Geriatric nurses lack knowledge of how to select appropriate foods for patients with dysphagia}

Timely early intervention measures can effectively reduce the incidence of dysphagia-related complications. As front-line medical workers, nurses implement nursing interventions that directly affect patient outcomes. Comprehensive clinical intervention measures for dysphagia include active treatment of the primary disease and accompanying symptoms, swallowing rehabilitation, dietary safety management, nursing intervention, and multidisciplinary team management. This study showed that only $53.56 \%$ of the nurses had accurate knowledge of dysphagia intervention measures, and only $44.88 \%$ of the nurses correctly answered the item "Patients with dysphagia are most susceptible to aspiration when they drink milk or lotus root powder soup or eat noodles or steamed bread". This result is consistent with the finding of Brady et al. (15) that $38 \%$ of nurses failed to choose appropriate foods for patients with dysphagia. In fact, the higher the viscosity of a fluid, the lower its flow rate in the mouth and pharynx, which give the patient more time to activate airway protection mechanisms, thereby reducing the risk of aspiration. This suggests that administrators need to provide standardized training for geriatric nurses on intervention measures for dysphagia, especially on the key points of eating safety, to improve the nurses' competency in the intervention and management of dysphagia.

\section{The attitudes and practices of nurses regarding the management of dysphagia in elderly patients are acceptable}

The main goal of dysphagia management is to prevent complications such as aspiration and malnutrition so that patients with dysphagia can obtain safe swallowing and, 
ultimately, a better quality of life. Nurses, as the medical workers who have the closest contact with patients, play an indispensable role in dysphagia screening, assessment, and intervention. The results of this study showed that $73.4 \%$ of the geriatric nurses believed that the identification of dysphagia should not be neglected in nursing work and that $86.6 \%$ thought that it is necessary to use relevant scales to screen elderly patients with a high risk of dysphagia in their daily work. However, only $65.1 \%$ of the geriatric nurses could provide a timely screening for dysphagia at admission. In addition, up to $91.5 \%$ of the geriatric nurses were willing to complete training on dysphagia-related knowledge and skills, and $39.9 \%$ indicated a strong willingness to undergo such training. This indicates that administrators should further strengthen the relevant trainings in their departments or throughout the hospital to meet nurses' learning needs regarding geriatric dysphagia-related knowledge and skills. The results of this survey showed that more than $80 \%$ of the nurses were able to educate the high-risk population/family members about dysphagia in their daily nursing work and could carefully observe patients' physical signs and provide eating guidance during meals. However, because the items on the questionnaire were objective, the results should be considered with caution. In the future, observations of nurses in a natural setting can be carried out to further explore the actual situation of dysphagia management among nurses. In addition, considering the problems regarding dysphagiarelated knowledge among, although nurses provide health education and eating guidance for patients with dysphagia, the scientificity and accuracy of the content of this education and guidance need to be improved.

\section{Difficulties encountered by nurses in caring for elderly people with dysphagia}

This study found that $89 \%$ of nurses believed that they lacked knowledge and skills related to dysphagia, and $71.87 \%$ of nurses lacked the awareness of caring for patients with dysphagia; $44.5 \%$ of nurses lack the time to take care of the elderly with dysphagia because they were busy with other tasks. In addition, $30.95 \%$ of nurses did not implement care measures because doctors did not issue medical orders related to the evaluation and care of dysphagia. It can be seen that nurses still have many difficulties in the process of caring for patients with dysphagia, which prompts us to strengthen the training of geriatric nurses' knowledge and skills in caring for dysphagia, and promote their active evaluation and detection of patients with dysphagia. This result also requires multidisciplinary interventions for the disease of dysphagia.

\section{Recommendations regarding dysphagia-related knowledge training for nurses}

At present, in some parts of China, nurses' knowledge and practice of swallowing disorders have been studied. Sun et al. investigated nurses from neurology departments (neurology department and neurosurgery department) in 18 hospitals in Beijing and found that the cognition of swallowing disorders of nurses was at a medium level, and the number of training, highest educational background, department and position were the main influencing factors (16). Nurses in three hospitals in Guangzhou (neurology, geriatrics, respiratory, cardiovascular and gastroenterology) had poor knowledge of dysphagia (17). A study of geriatric departments in Shenzhen found that nurses' knowledge of dysphagia was not high (18). Nurses in the neurology department are relatively familiar with swallowing disorders, which may be because swallowing disorders are more common in diseases such as stroke, cerebral palsy and brain trauma. This study showed that different titles, hospital levels, and past experiences with caring for elderly patients with dysphagia influenced the geriatric nurses' geriatric dysphagia-related knowledge scores, while age, years of work experience, and educational level were not among the main influencing factors. This may be because most geriatric nurses do not receive dysphagiaspecific information or training before or even they begin working, resulting in a lack of motivation and awareness regarding learning about dysphagia. In this study, geriatric nurses with higher titles and those who worked at higherlevel hospitals had better knowledge mastery, and knowledge and attitude have an impact on practice. Furthermore, good attitudes and practices require a wealth of knowledge as a foundation, and learning is an essential means of acquiring knowledge. Nurses with higher titles and those who work at higher-level hospitals have more opportunities to take training courses, participate in academic forums, obtain continuing education at other hospitals or institutions, and access richer learning resources and a wider range of learning pathways; consequently, their dysphagia-related knowledge scores were higher. Nurses with lower titles and those who work at lower-level hospitals have less access to medical resources, and updated knowledge becomes available to them more slowly; consequently, their dysphagia-related knowledge scores were lower. Nurses with experience caring 
for elderly patients with dysphagia will unconsciously learn information related to geriatric dysphagia on the job and apply their practical nursing experience to enrich their clinical practice. Hospital type and the duration since the last dysphagia training were factors that influenced nurses' practice and attitude scores, suggesting that administrators, especially those at nongeriatric hospitals, need to strengthen dysphagia-specific training and education in general practice and provide diversified training for geriatric nurses according to their characteristics. For nurses with lower titles, administrators should encourage participation in clinical teaching courses, provide them with more learning resources, strengthen management, and implement an assessment system so that these nurses can obtain geriatric dysphagia-related knowledge in a relatively short time. In addition, administrators should take fully advantage of nurses with higher professional titles. In clinical practice, new and experienced nurses can be paired, and each experienced nurse can teach a new nurse to further reduce knowledge and competency gaps between new and experienced nurses. Furthermore, different departments should regularly exchange nursing practice experiences to further promote nurses' mastery of geriatric dysphagia-related knowledge and improve their KAP to achieve overall improvement in the quality of the management of elderly patients with dysphagia at the departmental level or even the hospital level.

\section{Limitations of the study}

The geriatric nurses had acceptable practices and attitudes regarding the management of dysphagia in elderly patients, but their knowledge related to geriatric dysphagia, especially regarding risk factors such as drug use, recognizing aspiration, and selecting appropriate foods needs to be improved. Administrators should provide training that is targeted according to nurses' characteristics to improve dysphagia management and ensure the safety of elderly patients with dysphagia. On the basis of a literature review, this study used a scientific and objective questionnaire regarding dysphagia management knowledge to investigate the KAP of geriatric nurses toward dysphagia management and analyzed the factors that influenced their KAP. This study design was scientific and realistic as it considers the aging of the population in China and the great importance that the National Health Commission of China has attached to the health status of the elderly population. However, due to the lack of research time, experience, and resources, the study subjects were limited to geriatric nurses at secondary and tertiary hospitals in Sichuan, and consequently, the national representativeness of the sample was limited. This study was a cross-sectional study, and longitudinal studies are needed to further investigate the causal relationship between the independent variables and the dependent variables.

\section{Acknowledgments}

Funding: The research was supported by grants from the National Key R\&D Program of China (grant No. 2018YFC2001400), and the West China Nursing Discipline Development Special Fund Project, Sichuan University (grant No. HXHL19029).

\section{Footnote}

Reporting Checklist: The authors have completed the SURGE reporting checklist. Available at https://apm. amegroups.com/article/view/10.21037/apm-21-3672/rc

Data Sharing Statement: Available at https://apm.amegroups. com/article/view/10.21037/apm-21-3672/dss

Conflicts of Interest: All authors have completed the ICMJE uniform disclosure form (available at https://apm. amegroups.com/article/view/10.21037/apm-21-3672/coif). The authors have no conflicts of interest to declare.

Etbical Statement: The authors are accountable for all aspects of the work in ensuring that questions related to the accuracy or integrity of any part of the work are appropriately investigated and resolved. This study was approved by the Ethics Committee of the West China Hospital of Sichuan University (No. 1123, approved in 2021) and conducted in accordance with the Declaration of Helsinki (as revised in 2013). All nurses gave their informed consent.

Open Access Statement: This is an Open Access article distributed in accordance with the Creative Commons Attribution-NonCommercial-NoDerivs 4.0 International License (CC BY-NC-ND 4.0), which permits the noncommercial replication and distribution of the article with the strict proviso that no changes or edits are made and the original work is properly cited (including links to both the formal publication through the relevant DOI and the license). See: https://creativecommons.org/licenses/by-nc-nd/4.0/. 


\section{References}

1. Morris H. Dysphagia in the elderly--a management challenge for nurses. Br J Nurs 2006;15:558-62.

2. Aziz Q, Fass R, Gyawali CP, et al. Esophageal disorders. Gastroenterology 2016;150:1368-79.

3. Cabre M, Serra-Prat M, Palomera E, et al. Prevalence and prognostic implications of dysphagia in elderly patients with pneumonia. Age Ageing 2010;39:39-45.

4. Serra-Prat M, Palomera M, Gomez C, et al. Oropharyngeal dysphagia as a risk factor for malnutrition and lower respiratory tract infection in independently living older persons: a population-based prospective study. Age Ageing 2012;41:376-81.

5. Kawashima K, Motohashi Y, Fujishima I. Prevalence of dysphagia among community-dwelling elderly individuals as estimated using a questionnaire for dysphagia screening. Dysphagia 2004;19:266-71.

6. Kayser-Jones J, Pengilly K. Dysphagia among nursing home residents. Geriatr Nurs 1999;20:77-82; quiz 84.

7. Steele CM, Greenwood C, Ens I, et al. Mealtime difficulties in a home for the aged: not just dysphagia. Dysphagia 1997;12:43-50; discussion 51.

8. Lee A, Sitoh YY, Lieu PK, et al. Swallowing impairment and feeding dependency in the hospitalised elderly. Ann Acad Med Singap 1999;28:371-6.

9. Smith PA. Nutrition, hydration, and dysphagia in longterm care: Differing opinions on the effects of aspiration. J Am Med Dir Assoc 2006;7:545-9.

10. Mao X, Wang G, Chen R, et al. Constructing a

Cite this article as: Luo CR, Wei JY, Zhang XM. A multicenter cross-sectional survey of the knowledge, attitudes, and practices of geriatric nurses regarding dysphagia care. Ann Palliat Med 2022;11(1):16-25. doi: 10.21037/apm-21-3672 